\begin{tabular}{|c|c|c|}
\hline 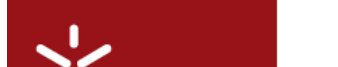 & CMAT & 1 \\
\hline - Reprositónill & Centro de Matemática da Universidade do Minho & -0 \\
\hline \multirow[t]{2}{*}{ Universidade do Minho } & Campus de Gualtar $4710-057$ Braga Portugal & $\begin{array}{l}\text { Universidade do Minho } \\
\text { Escola de Ciências }\end{array}$ \\
\hline & www.cmat.uminho.pt & Centro de Matemática \\
\hline
\end{tabular}

\title{
On a Variational Inequality for Incompressible Non-Newtonian Thick Flows
}

\author{
Fernando Miranda ${ }^{a}$ \\ José Francisco Rodrigues ${ }^{b}$ \\ ${ }^{a}$ CMAT and DMA, Universidade do Minho, Portugal \\ ${ }^{b}$ CMAF-CIO and F. Ciências, Universidade de Lisboa, Portugal
}

\section{Information}

Keywords:

Non-Newtonian flows

Thick fluids

Variational inequalities

Original publication:

Contemporary Mathematics 666,

305-316, 2016

DOI: $10.1090 /$ conm/666

http://www.ams.org

\begin{abstract}
In this work we extend the results on the existence, uniqueness and continuous dependence of strong solutions to a class of variational inequalities for incompressible non-Newtonian flows under the constraint of a variable maximum admissible shear rate. These fluids correspond to a limit case of shear-thickening viscosity, also called thick fluids, in which the solutions belong to a time dependent convex set with bounded deformation rate tensors. We also prove the existence of stationary solutions, which are the unique asymptotic limit of evolutionary flows in the case of sufficiently large viscosity.
\end{abstract}

\section{Dedicated to Hugo Beirão da Veiga on the occasion of his $70^{\text {th }}$ birthday}

\section{Introduction}

In a recent work [15] it has been shown that thickening a dilatant fluid, i.e. letting the power law in the relationship between shear rate and shear stress tend to infinity, leads to a new class of "thick fluids". This model has been motivated by an increasing interest in complex fluids showing abrupt raising of viscosity at certain critical levels of shear stress [1], namely in armor applications [9] as highlighted by the feature article in Physics Today [22].

Here we are interested in the constitutive law for the symmetric part of the velocity gradient $\mathbf{D} \boldsymbol{u}=$ $\frac{1}{2}\left(\nabla \boldsymbol{u}+\nabla \boldsymbol{u}^{T}\right)$ constrained by a variable positive threshold $\psi=\psi(x, t)$ :

$$
|\mathbf{D} \boldsymbol{u}(x, t)| \leq \psi(x, t) .
$$

If we denote by $\Omega \subset \mathbb{R}^{d}$ a bounded domain, with Lipschitz boundary $\partial \Omega, d \geq 2$ and $Q_{T}=\Omega \times(0, T)$, $T>0$, the constraint (1.1) for the velocity field $\boldsymbol{u}=\boldsymbol{u}(x, t)$ of the thick fluid divides, in general, the domain into two subregions $\{(x, t):|\mathbf{D} \boldsymbol{u}|<\psi\}$ and $\{(x, t):|\mathbf{D} \boldsymbol{u}|=\psi\}$. In the first subregion, i.e. strictly below the threshold, the classical system for the incompressible fluid for the velocity $\boldsymbol{u}$ and the pressure $\pi=\pi(x, t)$ holds:

$$
\begin{array}{r}
\partial_{t} \boldsymbol{u}-\operatorname{div}(\mathbf{S}-\boldsymbol{u} \otimes \boldsymbol{u})+\nabla \pi=\boldsymbol{f}, \\
\operatorname{div} \boldsymbol{u}=0,
\end{array}
$$


as it was observed in [15] for the stress tensor $\mathbf{S}$ given by

$$
\mathbf{S}=\mathbf{S}(\mathbf{D} \boldsymbol{u}):=\mu|\mathbf{D} \boldsymbol{u}|^{q-2} \mathbf{D} \boldsymbol{u},
$$

in the special Newtonian case $q=2$ with a constant viscosity $\mu>0$. Here $\partial_{t} \boldsymbol{u}=\frac{\partial \boldsymbol{u}}{\partial t}$ and $\operatorname{div}(\boldsymbol{u} \otimes \boldsymbol{u})=(\boldsymbol{u} \cdot \nabla) \boldsymbol{u}$ represents the usual convective term under the incompressibility condition (1.2b).

In this work we shall consider a more general non-Newtonian fluid by assuming that the stress tensor $\mathbf{S}: \Omega \times \mathbb{R}^{d^{2}} \longrightarrow \mathbb{R}^{d^{2}}$ is a Carathéodory function deriving from a scalar potential $\sigma$,

$$
\partial_{\boldsymbol{D}} \sigma(x, \boldsymbol{D})=\mathbf{S}(x, \boldsymbol{D}),
$$

such that $\mathbf{S}(x, \mathbf{0})=\mathbf{0}$ and, for $q>1$, satisfies the structure conditions

$$
\begin{gathered}
|\mathbf{S}(x, \boldsymbol{D})| \leq \kappa\left(1+|\boldsymbol{D}|^{q-2}\right)|\boldsymbol{D}|, \\
(\mathbf{S}(x, \boldsymbol{D})-\mathbf{S}(x, \boldsymbol{E})):(\boldsymbol{D}-\boldsymbol{E}) \geq\left\{\begin{array}{l}
\mu(|\boldsymbol{D}|+|\boldsymbol{E}|)^{q-2}|\boldsymbol{D}-\boldsymbol{E}|^{2} \text { if } q \leq 2, \\
\mu\left(1+|\boldsymbol{D}-\boldsymbol{E}|^{q-2}\right)|\boldsymbol{D}-\boldsymbol{E}|^{2} \text { if } q>2,
\end{array}\right.
\end{gathered}
$$

for given positive constants $\kappa, \mu$, for all $\boldsymbol{D}, \boldsymbol{E} \in \mathbb{R}^{d^{2}}$ and for a.e. $x \in \Omega$.

These assumptions include not only shear-thinning fluids with power law (1.3) with $1<q<2$ but also the Ladyzhenskaya model [7] for shear-thickening fluids of the type

$$
\mathbf{S}=\mathbf{S}(\mathbf{D} \boldsymbol{u}):=\mu_{*} \mathbf{D} \boldsymbol{u}+\mu|\mathbf{D} \boldsymbol{u}|^{q-2} \mathbf{D} \boldsymbol{u},
$$

with $q>2$. In the case $q=2$ we may also include non-Newtonian fluids

$$
\mathbf{S}(x, \boldsymbol{D})=s(x,|\boldsymbol{D}|) \boldsymbol{D}
$$

of potential type with

$$
\sigma(x, \boldsymbol{D})=\int_{0}^{|\boldsymbol{D}|} \tau s(x, \tau) d \tau,
$$

provided the function $s: \Omega \times \mathbb{R}^{+} \longrightarrow \mathbb{R}^{+}$satisfies the condition

$$
0<\mu \leq \frac{\partial}{\partial \tau}(\tau s(x, \tau)) \leq \kappa, \forall \tau \geq 0 \text {, a.e. } x \in \Omega .
$$

To complete the mathematical formulation we shall choose the usual Dirichlet and initial boundary conditions

$$
\boldsymbol{u}=\mathbf{0} \quad \text { on } \partial \Omega \times(0, T), \quad \boldsymbol{u}(0)=\boldsymbol{u}_{0} \text { in } \Omega .
$$

Several variants of generalized Newtonian fluids of these type without the constraint (1.1) have been considered in the mathematical literature (see, for instance, the books [8], [10], [11] or the survey [12]). For instance, the existence of weak solutions for shear-thinning fluids has been shown for $d=3$ up to $p>\frac{6}{5}$ in [4] and higher regularity up to the boundary for shear-thickening fluids has been obtained in [2], where additional references may be found.

The special case of the constraint $\psi=1$ has been considered in the the earlier work [19] to obtain the existence of weak solutions for non-Newtonian flows with rigid bodies in which the inhomogeneous viscosity is a solution of a transport equation and in the recent work [3] for the steady-state Stokes system with numerical examples.

It is well known that gradient type constraints arise in the mathematical formulation of several problems in Mechanics and in Physics, namely in critical state models of plasticity (the elastoplastic torsion problem), superconductivity (magnetization of type-II superconductors), geophysics descriptions (sandpile growth or formation of network of lakes and rivers), leading to variational or quasi-variational inequalities (see, for instance, [14], [16], [13] or [17] and their references). In fluid mechanics, a different kind of variational inequalities arise also in certain limits of non-Newtonian flows, namely in the case of Bingham fluids, [5], [21], which correspond also to flows with two phases (rigid if $|\mathbf{D} \boldsymbol{u}|=0$ for $|\mathbf{S}| \leq s_{*}$ and fluid for $|\mathbf{S}|>s_{*}>0$ ) although of different nature.

In the next section we introduce the variational inequality associated with the constraint (1.1) and we show the existence and uniqueness of a strong solution by using a penalization method as in [15], similarly to [18] and [13]. We complete the well-posedness of the problem by showing its continuous dependence with respect to the data, including the threshold $\psi$. Finally, for sufficiently large viscosities, we also show the asymptotic stabilization as $t \rightarrow \infty$ towards the steady-state solution. 


\section{Existence of strong variational solutions}

In order to introduce the variational inequality formulation we set the following notations:

$$
\begin{aligned}
\mathbb{J}(\Omega) & :=\left\{\boldsymbol{\varphi} \in \mathscr{C}_{0}^{\infty}(\Omega)^{d}: \operatorname{div} \boldsymbol{\varphi}=0\right\}, \\
\mathbb{L}_{\sigma}^{r}(\Omega) & :=\text { closure of } \mathbb{J} \text { in } \boldsymbol{L}^{r}(\Omega), \\
\mathbb{V}_{r}(\Omega) & :=\text { closure of } \mathbb{J} \text { in } \boldsymbol{W}^{1, r}(\Omega),
\end{aligned}
$$

where $W^{1, r}(\Omega)$ is the Sobolev space with $1<r<\infty, \boldsymbol{L}^{r}(\Omega)=L^{r}(\Omega)^{d}$ and $\boldsymbol{W}^{1, r}(\Omega)=W^{1, r}(\Omega)^{d}$.

Let $\psi=\psi(x, t): Q_{T} \longrightarrow \mathbb{R}^{+}$be a given function, such that,

$$
\psi \geq \psi_{*}>0 \quad \text { and } \quad \psi \in W^{1, \infty}\left(0, T ; L^{\infty}(\Omega)\right)
$$

and let us define the closed convex set

$$
\mathbb{K}(t):=\left\{\boldsymbol{w} \in \mathbb{V}_{r}(\Omega):|\mathbf{D} \boldsymbol{w}| \leq \psi(\cdot, t), \text { a.e. in } \Omega\right\},
$$

which is nonempty and, in fact, $\mathbb{K}(t) \subset \bigcap_{1<r<\infty} \mathbb{V}_{r}(\Omega) \subset \boldsymbol{L}^{\infty}(\Omega)$, for all $t \in(0, T)$.

Theorem 2.1. Assuming that

$$
\boldsymbol{f} \in \boldsymbol{L}^{q^{\prime} \vee 2}\left(Q_{T}\right) \quad \text { and } \quad \boldsymbol{u}_{0} \in \mathbb{K}(0),
$$

with $q^{\prime} \vee 2=\max (q /(q-1), 2)$, there exists a unique solution

$$
\left.\boldsymbol{u} \in L^{\infty}\left(0, T ; \bigcap_{1<r<\infty} \mathbb{V}_{r}(\Omega)\right) \cap H^{1}\left(0, T ; \mathbb{L}_{\sigma}^{2}(\Omega)\right)\right)
$$

to the following variational inequality for the incompressible non-Newtonian thick flow problem:

$$
\boldsymbol{u}(t) \in \mathbb{K}(t) \text { for } t \in(0, T), \boldsymbol{u}(0)=\boldsymbol{u}_{0},
$$

$$
\begin{aligned}
\int_{\Omega} \partial_{t} \boldsymbol{u} \cdot(\boldsymbol{w}-\boldsymbol{u})+\int_{\Omega} \mathbf{S}(\mathbf{D} \boldsymbol{u}): \mathbf{D}(\boldsymbol{w}-\boldsymbol{u})-\int_{\Omega}(\boldsymbol{u} \otimes \boldsymbol{u}): \nabla(\boldsymbol{w}-\boldsymbol{u}) & \\
& \geq \int_{\Omega} \boldsymbol{f} \cdot(\boldsymbol{w}-\boldsymbol{u}), \quad \forall \boldsymbol{w} \in \mathbb{K}(t), \text { a.e. } t \in(0, T),
\end{aligned}
$$

Proof. The proof of the existence uses a penalization approach as in $[18,13,15]$.

For a positive parameter $\varepsilon<1$ let $\kappa_{\varepsilon}: \mathbb{R} \rightarrow \mathbb{R}_{0}^{+}$be the continuous and increasing function such that

$$
\kappa_{\varepsilon}(s):= \begin{cases}0 & \text { if } s \leq 0 \\ e^{\frac{s}{\varepsilon}}-1 & \text { if } s \geq 0\end{cases}
$$

and let us consider the monotone and strictly coercive operator

$$
\mathbf{S}_{\varepsilon}(x, \boldsymbol{D}):=\mathbf{S}(x, \boldsymbol{D})+\kappa_{\varepsilon}\left(|\boldsymbol{D}|^{q}-\psi^{q}\right)|\boldsymbol{D}|^{q-2} \boldsymbol{D} .
$$

For each $\varepsilon$, using Galerkin method and the estimates below(see [10]) we may guarantee the existence and uniqueness of

$$
\boldsymbol{u}_{\varepsilon} \in L^{r}\left(0, T ; \bigcap_{1<r<\infty} \mathbb{V}_{r}(\Omega)\right) \cap H^{1}\left(0, T ; \mathbb{L}_{\sigma}^{2}(\Omega)\right)
$$

solving the penalized problem

$$
\begin{aligned}
\int_{\Omega} \partial_{t} \boldsymbol{u}_{\varepsilon} \cdot \boldsymbol{\varphi}+\int_{\Omega} \mathbf{S}_{\varepsilon}\left(\mathbf{D} \boldsymbol{u}_{\varepsilon}\right): \mathbf{D} \boldsymbol{\varphi}-\int_{\Omega}\left(\boldsymbol{u}_{\varepsilon} \otimes \boldsymbol{u}_{\varepsilon}\right): \nabla \boldsymbol{\varphi} & =\int_{\Omega} \boldsymbol{f} \cdot \boldsymbol{\varphi}, \quad \forall \boldsymbol{\varphi} \in \mathbb{V}_{q}, \text { a.e. } t \in(0, T), \\
\boldsymbol{u}_{\varepsilon}(0) & =\boldsymbol{u}_{0} .
\end{aligned}
$$


Indeed, the solutions $\boldsymbol{u}_{\varepsilon}$ satisfy the following a priori estimates, independently of $\varepsilon$ :

$$
\begin{gathered}
\left\|\kappa_{\varepsilon}\left(\left|\mathbf{D} \boldsymbol{u}_{\varepsilon}\right|^{q}-\psi^{p}\right)\left|\mathbf{D} \boldsymbol{u}_{\varepsilon}\right|^{q}\right\|_{L^{1}\left(Q_{T}\right)} \leq C_{0} \\
\left\|\kappa_{\varepsilon}\left(\left|\mathbf{D} \boldsymbol{u}_{\varepsilon}\right|^{q}-\boldsymbol{\psi}^{q}\right)\right\|_{L^{1}\left(Q_{T}\right)} \leq C_{1}, \\
\left\|\mathbf{D} \boldsymbol{u}_{\varepsilon}\right\|_{L^{r}\left(Q_{T}\right)} \leq C_{2}, \quad \forall 1 \leq r<\infty \\
\left\|\partial_{t} \boldsymbol{u}_{\varepsilon}\right\|_{\boldsymbol{L}^{2}\left(Q_{T}\right)} \leq C_{3} .
\end{gathered}
$$

Testing equation (2.5a) with $\varphi=\boldsymbol{u}_{\varepsilon}$ the estimate (2.6) follows at once, obtaining in addition the usual energy estimates

$$
\left\|\boldsymbol{u}_{\varepsilon}\right\|_{L^{\infty}\left(0, T ; \boldsymbol{L}^{2}(\Omega)\right)} \leq C_{0} \quad \text { and } \quad\left\|\mathbf{D} \boldsymbol{u}_{\varepsilon}\right\|_{\boldsymbol{L}^{q}\left(Q_{T}\right)} \leq C_{0} .
$$

It is immediate to conclude that

$$
\int_{Q_{T}} \kappa_{\varepsilon}\left(\left|\mathbf{D} \boldsymbol{u}_{\varepsilon}\right|^{q}-\psi^{q}\right)\left(\left|\mathbf{D} \boldsymbol{u}_{\varepsilon}\right|^{q}-\psi^{q}\right) \geq 0
$$

since, by construction, $\kappa_{\epsilon}(s)=0$ for $s \leq 0$ and $\kappa_{\epsilon}(s)>0$ for $s>0$.

Recalling that $\psi_{*}$ is a positive lower bound to the constraint function $\psi$ and taking into account (2.10), we get

$$
\begin{aligned}
& \int_{Q_{T}} \kappa_{\varepsilon}\left(\left|\mathbf{D} \boldsymbol{u}_{\varepsilon}\right|^{q}-\psi^{q}\right) \leq \int_{Q_{T}} \kappa_{\varepsilon}\left(\left|\mathbf{D} \boldsymbol{u}_{\varepsilon}\right|^{q}-\psi^{q}\right) \frac{\psi^{q}}{\psi_{*}^{q}} \\
&=\frac{1}{\psi_{*}^{q}}\left(\int_{Q_{T}} \kappa_{\varepsilon}\left(\left|\mathbf{D} \boldsymbol{u}_{\varepsilon}\right|^{q}-\psi^{q}\right)\left(\psi^{q}-\left|\mathbf{D} \boldsymbol{u}_{\varepsilon}\right|^{q}\right)+\int_{Q_{T}} \kappa_{\varepsilon}\left(\left|\mathbf{D} \boldsymbol{u}_{\varepsilon}\right|^{q}-\psi^{q}\right)\left|\mathbf{D} \boldsymbol{u}_{\varepsilon}\right|^{q}\right) \\
& \quad \leq \frac{1}{\psi_{*}^{q}} \int_{Q_{T}} \kappa_{\varepsilon}\left(\left|\mathbf{D} \boldsymbol{u}_{\varepsilon}\right|^{q}-\psi^{q}\right)\left|\mathbf{D} \boldsymbol{u}_{\varepsilon}\right|^{q}
\end{aligned}
$$

so, estimate (2.7) follows from (2.6).

Observing that, for $s \geq \varepsilon, \kappa_{\varepsilon}(s)=e^{\frac{s}{\varepsilon}}-1 \geq \frac{s^{\eta}}{\eta ! \varepsilon^{\eta}}$, defining

$$
D_{\varepsilon}:=\left\{(x, t) \in Q_{T}:\left|\mathbf{D} \boldsymbol{u}_{\varepsilon}\right|^{q}-\psi^{q} \geq \varepsilon\right\},
$$

from the estimate (2.6) we have

$$
C_{0} \geq \int_{Q_{T}} \kappa_{\varepsilon}\left(\left|\mathbf{D} \boldsymbol{u}_{\varepsilon}\right|^{q}-\psi^{q}\right)\left|\mathbf{D} \boldsymbol{u}_{\varepsilon}\right|^{q} \geq \int_{D_{\varepsilon}} \frac{\left(\left|\mathbf{D} \boldsymbol{u}_{\varepsilon}\right|^{q}-\psi^{q}\right)^{\eta}}{\eta ! \varepsilon^{\eta}}\left|\mathbf{D} \boldsymbol{u}_{\varepsilon}\right|^{q}
$$

enabling us to conclude (2.8) by arguing as in Lemma 4 of [16] (see also the proof of Proposition 3.14 of [17]).

Choosing $r>2$, the estimate (2.8) allows the control of the convection term:

$$
\int_{Q_{T}}\left(\boldsymbol{u}_{\varepsilon} \otimes \boldsymbol{u}_{\varepsilon}\right) \cdot \nabla \partial_{t} \boldsymbol{u}_{\varepsilon}=\int_{Q_{T}}\left(\boldsymbol{u}_{\varepsilon} \cdot \nabla\right) \boldsymbol{u}_{\varepsilon} \cdot \partial_{t} \boldsymbol{u}_{\varepsilon} \leq C_{r}\left\|\boldsymbol{u}_{\varepsilon}\right\|_{L^{\frac{2 r}{r-2}}\left(Q_{T}\right)}^{2}\left\|\mathbf{D} \boldsymbol{u}_{\varepsilon}\right\|_{L^{r}\left(Q_{T}\right)}^{2}+\frac{1}{3}\left\|\partial_{t} \boldsymbol{u}_{\varepsilon}\right\|_{L^{2}\left(Q_{T}\right)}^{2} .
$$

Letting formally $\boldsymbol{\varphi}=\partial_{t} \boldsymbol{u}_{\varepsilon}$ in (2.5a), we have

$$
\begin{aligned}
\int_{\Omega}\left|\partial_{t} \boldsymbol{u}_{\varepsilon}\right|^{2}+\int_{\Omega} \mathbf{S}\left(\mathbf{D} \boldsymbol{u}_{\varepsilon}\right): \partial_{t} \mathbf{D} \boldsymbol{u}_{\varepsilon}+\int_{\Omega} \kappa_{\varepsilon}\left(\left|\mathbf{D} \boldsymbol{u}_{\varepsilon}\right|^{q}-\psi^{p}\right)\left|\mathbf{D} \boldsymbol{u}_{\varepsilon}\right|^{q-2} \mathbf{D} \boldsymbol{u}_{\varepsilon}: \partial_{t} \mathbf{D} \boldsymbol{u}_{\varepsilon} & =\int_{\Omega} \boldsymbol{f} \cdot \partial_{t} \boldsymbol{u}_{\varepsilon}+\int_{\Omega}\left(\boldsymbol{u}_{\varepsilon} \otimes \boldsymbol{u}_{\varepsilon}\right): \nabla \partial_{t} \boldsymbol{u}_{\varepsilon} .
\end{aligned}
$$

Setting $\phi_{\varepsilon}(s)=\int_{0}^{s} \kappa_{\varepsilon}(\tau) d \tau$ and, from (1.4), recalling that $\mathbf{S}$ is potential, integrating the last expression up to time $t \in(0, T]$, we obtain

$$
\begin{aligned}
\int_{Q_{t}}\left|\partial_{t} \boldsymbol{u}_{\varepsilon}\right|^{2}+\int_{\Omega} \sigma\left(\mathbf{D} \boldsymbol{u}_{\varepsilon}(t)\right)+\frac{1}{q} \int_{\Omega} \phi_{\varepsilon}\left(\left|\mathbf{D} \boldsymbol{u}_{\varepsilon}(t)\right|^{q}-\psi(t)^{q}\right)+\int_{Q_{t}} \kappa_{\varepsilon}\left(\left|\mathbf{D} \boldsymbol{u}_{\varepsilon}\right|^{q}-\psi^{q}\right) \psi^{q-1} \partial_{t} \psi & \\
& =\int_{Q_{t}} \boldsymbol{f} \cdot \partial_{t} \boldsymbol{u}_{\varepsilon}+\int_{Q_{t}}\left(\boldsymbol{u}_{\varepsilon} \otimes \boldsymbol{u}_{\varepsilon}\right): \nabla \partial_{t} \boldsymbol{u}_{\varepsilon}+\int_{\Omega} \sigma\left(\mathbf{D} \boldsymbol{u}_{0}\right) .
\end{aligned}
$$


Applying Hölder inequality and taking into account (2.11) we obtain

$$
\begin{aligned}
\left\|\partial_{t} \boldsymbol{u}_{\varepsilon}\right\|_{\boldsymbol{L}^{2}\left(Q_{T}\right)}^{2} \leq C\left(\|\boldsymbol{f}\|_{\boldsymbol{L}^{2}\left(Q_{T}\right)}^{2}+\left\|\boldsymbol{u}_{\varepsilon}\right\|_{\boldsymbol{L}^{\frac{2 r}{r-2}}\left(Q_{T}\right)}^{2}\left\|\mathbf{D} \boldsymbol{u}_{\varepsilon}\right\|_{\boldsymbol{L}^{r}\left(Q_{T}\right)}^{2}\right. & \\
& \left.+\left\|\kappa_{\varepsilon}\left(\left|\mathbf{D} \boldsymbol{u}_{\varepsilon}\right|^{q}-\psi^{q}\right)\right\|_{L^{1}\left(Q_{T}\right)}\left\|\psi^{q-1}\right\|_{L^{\infty}\left(Q_{T}\right)}\left\|\partial_{t} \psi\right\|_{L^{\infty}\left(Q_{T}\right)}+1\right)
\end{aligned}
$$

which proves (2.9).

Hence, by compactness (see [20]), there exists

$$
\boldsymbol{u} \in L^{r}\left(0, T ; \bigcap_{1<r<\infty} \mathbb{V}_{r}(\Omega)\right) \cap H^{1}\left(0, T ; \mathbb{L}_{\sigma}^{2}(\Omega)\right), \text { with } \boldsymbol{u}(0)=\boldsymbol{u}_{0},
$$

such that, at least for a subsequence $\varepsilon \rightarrow 0$,

$$
\begin{aligned}
\boldsymbol{u}_{\varepsilon} \rightarrow \boldsymbol{u} \quad \text { in } \mathscr{C}\left(\bar{Q}_{T}\right), \\
\mathbf{D} \boldsymbol{u}_{\varepsilon} \rightarrow \mathbf{D} \boldsymbol{u} \quad \text { in } \boldsymbol{L}^{r}\left(Q_{T}\right) \text {-weak, } \forall 1 \leq r<\infty, \\
\partial_{t} \boldsymbol{u}_{\varepsilon} \rightarrow \partial_{t} \boldsymbol{u} \quad \text { in } \boldsymbol{L}^{2}\left(Q_{T}\right) \text {-weak. }
\end{aligned}
$$

The limit function $\boldsymbol{u}(t)$ belongs to the convex set $\mathbb{K}(t)$, for a.e. $t \in(0, T)$. Indeed, splitting $Q_{T}$ in the sets

$$
\begin{aligned}
& A_{\varepsilon}:=\left\{(x, t) \in Q_{T}:\left|\mathbf{D} \boldsymbol{u}_{\varepsilon}(x, t)\right|^{q}-\psi(x, t)^{q}<\sqrt{\varepsilon}\right\}, \\
& B_{\varepsilon}:=\left\{(x, t) \in Q_{T}:\left|\mathbf{D} \boldsymbol{u}_{\varepsilon}(x, t)\right|^{q}-\psi(x, t)^{q} \geq \sqrt{\varepsilon}\right\},
\end{aligned}
$$

we observe that

$$
\left|B_{\varepsilon}\right|=\int_{B_{\varepsilon}} 1 \leq \int_{B_{\varepsilon}} \frac{\kappa_{\varepsilon}\left(\left|\mathbf{D} \boldsymbol{u}_{\varepsilon}\right|^{q}-\psi^{q}\right)}{e^{\frac{1}{\sqrt{\varepsilon}-1}}} \leq \frac{C_{1}}{e^{\frac{1}{\sqrt{\varepsilon}}}-1} \underset{\varepsilon \rightarrow 0}{\longrightarrow} 0,
$$

and we conclude that $|\mathbf{D} \boldsymbol{u}(t)| \leq \psi(t)$ a.e. in $Q_{T}$ from

$$
\begin{aligned}
\int_{Q_{T}}\left(\left|\mathbf{D} \boldsymbol{u}_{\varepsilon}\right|^{q}-\psi^{q}\right)^{+} & \leq \liminf _{\varepsilon \rightarrow 0} \int_{Q_{T}}\left(\left|\mathbf{D} \boldsymbol{u}_{\varepsilon}\right|^{q}-\psi^{q}\right) \vee \sqrt{\varepsilon} \\
& \leq \liminf _{\varepsilon \rightarrow 0} \int_{A_{\varepsilon}} \sqrt{\varepsilon}+\liminf _{\varepsilon \rightarrow 0} \int_{B_{\varepsilon}}\left(\left|\mathbf{D} \boldsymbol{u}_{\varepsilon}\right|^{q}-\psi^{q}\right) \\
& \leq \liminf _{\varepsilon \rightarrow 0} \int_{Q_{T}}\left(\left|\mathbf{D} \boldsymbol{u}_{\varepsilon}\right|^{q}-\psi^{q}\right) \chi_{B_{\varepsilon}} \\
& \leq \lim _{\varepsilon \rightarrow 0}\left\|\left|\mathbf{D} \boldsymbol{u}_{\varepsilon}\right|^{q}-\psi^{q}\right\|_{L^{1}\left(Q_{T}\right)}\left|B_{\varepsilon}\right| \underset{\varepsilon \rightarrow 0}{\longrightarrow} 0 .
\end{aligned}
$$

It remains to prove that $\boldsymbol{u}$ solves de variational inequality (2.4). For $\boldsymbol{w}=\boldsymbol{w}(t) \in \mathbb{K}(t)$, testing equation (2.5a) with $\boldsymbol{w}-\boldsymbol{u}_{\varepsilon}$ we obtain

$$
\int_{\Omega} \partial_{t} \boldsymbol{u}_{\varepsilon} \cdot\left(\boldsymbol{w}-\boldsymbol{u}_{\varepsilon}\right)+\int_{\Omega} \mathbf{S}_{\varepsilon}\left(\mathbf{D} \boldsymbol{u}_{\varepsilon}\right): \mathbf{D}\left(\boldsymbol{w}-\boldsymbol{u}_{\varepsilon}\right)-\int_{\Omega}\left(\boldsymbol{u}_{\varepsilon} \otimes \boldsymbol{u}_{\varepsilon}\right): \nabla\left(\boldsymbol{w}-\boldsymbol{u}_{\varepsilon}\right)=\int_{\Omega} \boldsymbol{f} \cdot\left(\boldsymbol{w}-\boldsymbol{u}_{\varepsilon}\right) .
$$

Since $\mathbf{S}_{\varepsilon}$ is a monotone operator, we have

$$
\begin{aligned}
\mathbf{S}_{\varepsilon}\left(\mathbf{D} \boldsymbol{u}_{\varepsilon}\right): \mathbf{D}\left(\boldsymbol{w}-\boldsymbol{u}_{\varepsilon}\right) \leq \mathbf{S}(\mathbf{D} \boldsymbol{w}) & : \mathbf{D}\left(\boldsymbol{w}-\boldsymbol{u}_{\varepsilon}\right) \\
& +\kappa_{\varepsilon}\left(|\mathbf{D} \boldsymbol{w}|^{q}-\psi^{p}\right)|\mathbf{D} \boldsymbol{w}|^{q-2} \mathbf{D} \boldsymbol{w}: \mathbf{D}\left(\boldsymbol{w}-\boldsymbol{u}_{\varepsilon}\right)=\mathbf{S}(\mathbf{D} \boldsymbol{w}): \mathbf{D}\left(\boldsymbol{w}-\boldsymbol{u}_{\varepsilon}\right)
\end{aligned}
$$

and so

$$
\int_{\Omega} \partial_{t} \boldsymbol{u}_{\varepsilon} \cdot\left(\boldsymbol{w}-\boldsymbol{u}_{\varepsilon}\right)+\int_{\Omega} \mathbf{S}(\mathbf{D} \boldsymbol{w}): \mathbf{D}\left(\boldsymbol{w}-\boldsymbol{u}_{\varepsilon}\right)-\int_{\Omega}\left(\boldsymbol{u}_{\varepsilon} \otimes \boldsymbol{u}_{\varepsilon}\right): \nabla\left(\boldsymbol{w}-\boldsymbol{u}_{\varepsilon}\right) \geq \int_{\Omega} \boldsymbol{f} \cdot\left(\boldsymbol{w}-\boldsymbol{u}_{\varepsilon}\right) .
$$

Passing to the limit in $\varepsilon$ we obtain, using Minty's Lemma,

$$
\int_{\Omega} \partial_{t} \boldsymbol{u} \cdot(\boldsymbol{w}-\boldsymbol{u})+\int_{\Omega} \mathbf{S}(\mathbf{D} \boldsymbol{u}): \mathbf{D}(\boldsymbol{w}-\boldsymbol{u})-\int_{\Omega}(\boldsymbol{u} \otimes \boldsymbol{u}): \nabla(\boldsymbol{w}-\boldsymbol{u}) \geq \int_{\Omega} \boldsymbol{f} \cdot(\boldsymbol{w}-\boldsymbol{u}) .
$$


In order to prove the uniqueness of the solution to problem (2.4) it is crucial the control of the convective terms of any two solutions $\boldsymbol{u}_{1}$ and $\boldsymbol{u}_{2}$, in terms of their difference $\boldsymbol{w}=\boldsymbol{u}_{1}-\boldsymbol{u}_{2}$. In fact, by regularity we have that $\boldsymbol{u}_{1}$ and $\boldsymbol{u}_{2}$ are in fact in $\boldsymbol{L}^{\infty}\left(Q_{T}\right)$ and so, using Korn inequality,

$$
\begin{aligned}
\left|\int_{\Omega}\left(\boldsymbol{u}_{1} \otimes \boldsymbol{u}_{1}-\boldsymbol{u}_{2} \otimes \boldsymbol{u}_{2}\right): \nabla \boldsymbol{w}\right| \leq \int_{\Omega}\left|\left(\boldsymbol{u}_{1} \otimes \boldsymbol{w}+\boldsymbol{w} \otimes \boldsymbol{u}_{2}\right): \nabla \boldsymbol{w}\right| \\
\quad \leq c_{1}\|\boldsymbol{w}\|_{\boldsymbol{L}^{2}(\Omega)}\|\nabla \boldsymbol{w}\|_{\boldsymbol{L}^{2}(\Omega)} \leq c_{2}\|\boldsymbol{w}\|_{\boldsymbol{L}^{2}(\Omega)}\|\mathbf{D} \boldsymbol{w}\|_{\boldsymbol{L}^{2}(\Omega)} .
\end{aligned}
$$

Observing that $\boldsymbol{u}_{1}$ and $\boldsymbol{u}_{2}$ satisfy for a.e. $t$ the inequality

$$
\int_{\Omega} \partial_{t} \boldsymbol{w} \cdot \boldsymbol{w}+\int_{\Omega}\left(\mathbf{S}\left(\mathbf{D} \boldsymbol{u}_{1}\right)-\mathbf{S}\left(\mathbf{D} \boldsymbol{u}_{2}\right)\right): \mathbf{D} \boldsymbol{w} \leq \int_{\Omega}\left(\boldsymbol{u}_{1} \otimes \boldsymbol{w}+\boldsymbol{w} \otimes \boldsymbol{u}_{2}\right): \nabla \boldsymbol{w},
$$

taking into account (1.5b) and that $\mathbf{D} \boldsymbol{u}_{1}$ and $\mathbf{D} \boldsymbol{u}_{2}$ are in $\boldsymbol{L}^{\infty}\left(Q_{T}\right)$, by (2.12) we obtain,

$$
\frac{d}{d t}\|\boldsymbol{w}\|_{\boldsymbol{L}^{2}(\Omega)}^{2}+c_{3}\|\mathbf{D} \boldsymbol{w}\|_{\boldsymbol{L}^{2}(\Omega)}^{2} \leq c_{2}\|\boldsymbol{w}\|_{\boldsymbol{L}^{2}(\Omega)}\|\mathbf{D} \boldsymbol{w}\|_{\boldsymbol{L}^{2}(\Omega)}
$$

and therefore we get

$$
\frac{d}{d t}\|\boldsymbol{w}\|_{\boldsymbol{L}^{2}(\Omega)}^{2} \leq c\|\boldsymbol{w}\|_{\boldsymbol{L}^{2}(\Omega)}^{2} .
$$

Observing that $\boldsymbol{w}(0)=\mathbf{0}$, Gronwall's inequality implies the uniqueness of the solution of (2.4).

\section{Continuous dependence and asymptotic stabilization}

In fact, the properties of the strong solutions, the strict coercivity of $\mathbf{S}$ and an argument of [18] concerning the continuity of the convex set (2.2) with respect to the threshold $\psi$ allows us to obtain an estimate on the continuous dependence with respect of the data.

Theorem 3.1. Let, for $i=1,2, \boldsymbol{u}_{i}$ denote the solution to the variational inequality (2.4) with data $\psi_{i}$, satisfying (2.1), $\boldsymbol{f}_{i}$ and $\boldsymbol{u}_{i_{0}}$, as in (2.3). Then, there exists a positive constant $C=C(T)$ such that

$$
\begin{aligned}
\left\|\boldsymbol{u}_{1}-\boldsymbol{u}_{2}\right\|_{L^{\infty}\left(0, T, \boldsymbol{L}^{2}(\Omega)\right)}^{2}+\| \mathbf{D} & \left(\boldsymbol{u}_{1}-\boldsymbol{u}_{2}\right) \|_{\boldsymbol{L}^{2}\left(Q_{T}\right)}^{2} \\
& \leq C\left(\left\|\boldsymbol{f}_{1}-\boldsymbol{f}_{2}\right\|_{\boldsymbol{L}^{2}\left(Q_{T}\right)}^{2}+\left\|\boldsymbol{u}_{1_{0}}-\boldsymbol{u}_{2_{0}}\right\|_{\boldsymbol{L}^{2}\left(Q_{T}\right)}^{2}+\left\|\psi_{1}-\psi_{2}\right\|_{L^{\infty}\left(Q_{T}\right)}\right) .
\end{aligned}
$$

Proof. Let $\psi_{i}, i=1,2$, be given threshold functions satisfying (2.1) and denote by $\mathbb{K}_{i}(t)$ the corresponding convex sets defined in (2.2). For $i, j=1,2, i \neq j$, and for any $\boldsymbol{u}_{i} \in \mathbb{K}_{i}(t)$, there exists $\boldsymbol{u}_{j i} \in \mathbb{K}_{j}(t)$ such that

$$
\left\|\mathbf{D}\left(\boldsymbol{u}_{i}(t)-\boldsymbol{u}_{j i}(t)\right)\right\|_{\boldsymbol{L}^{q}(\Omega)} \leq C \beta(t) \text {, where } \beta(t):=\mid \psi_{i}(t)-\psi_{j}(t) \|_{L^{\infty}(\Omega)} .
$$

Indeed, following [18], it is enough to choose

$$
\boldsymbol{u}_{j i}(t):=\frac{\psi_{*} \boldsymbol{u}_{i}(t)}{\psi_{*}+\beta(t)} \quad \text { and } \quad C \geq \frac{1}{\psi_{*}}\left\|\mathbf{D} \boldsymbol{u}_{i}(t)\right\|_{\boldsymbol{L}^{q}(\Omega)} .
$$

Considering, for $i=1,2$, the solution $\boldsymbol{u}_{i}$ of the variational inequality (2.4) associated to the constraint $\psi_{i}$, using $\boldsymbol{u}_{i j}, j=1,2, j \neq i$, as test function, we have

$$
\int_{\Omega} \partial_{t} \boldsymbol{u}_{i} \cdot\left(\boldsymbol{u}_{i}-\boldsymbol{u}_{i j}\right)+\int_{\Omega} \mathbf{S}\left(\mathbf{D} \boldsymbol{u}_{i}\right): \mathbf{D}\left(\boldsymbol{u}_{i}-\boldsymbol{u}_{i j}\right) \leq \int_{\Omega}\left(\boldsymbol{u}_{i} \otimes \boldsymbol{u}_{i}\right): \nabla\left(\boldsymbol{u}_{i}-\boldsymbol{u}_{i j}\right)+\int_{\Omega} \boldsymbol{f}_{i} \cdot\left(\boldsymbol{u}_{i}-\boldsymbol{u}_{i j}\right)
$$

and so

$$
\begin{aligned}
\int_{\Omega} \partial_{t} \boldsymbol{u}_{i} \cdot\left(\boldsymbol{u}_{i}-\boldsymbol{u}_{j}\right)+ & \int_{\Omega} \mathbf{S}\left(\mathbf{D} \boldsymbol{u}_{i}\right): \mathbf{D}\left(\boldsymbol{u}_{i}-\boldsymbol{u}_{j}\right) \\
\leq & \int_{\Omega}\left(\boldsymbol{u}_{i} \otimes \boldsymbol{u}_{i}\right): \nabla\left(\boldsymbol{u}_{i}-\boldsymbol{u}_{j}\right)+\int_{\Omega} \boldsymbol{f}_{i} \cdot\left(\boldsymbol{u}_{i}-\boldsymbol{u}_{j}\right)+\int_{\Omega} \partial_{t} \boldsymbol{u}_{i} \cdot\left(\boldsymbol{u}_{i j}-\boldsymbol{u}_{j}\right) \\
& \quad+\int_{\Omega} \mathbf{S}\left(\mathbf{D} \boldsymbol{u}_{i}\right): \mathbf{D}\left(\boldsymbol{u}_{i j}-\boldsymbol{u}_{j}\right)+\int_{\Omega}\left(\boldsymbol{u}_{i} \otimes \boldsymbol{u}_{i}\right): \nabla\left(\boldsymbol{u}_{j}-\boldsymbol{u}_{i j}\right)+\int_{\Omega} \boldsymbol{f}_{i} \cdot\left(\boldsymbol{u}_{j}-\boldsymbol{u}_{i j}\right) .
\end{aligned}
$$


Adding inequalities we obtained in the former expression to $(i, j)=(1,2)$ and $(i, j)=(2,1)$, denoting $\boldsymbol{w}=\boldsymbol{u}_{1}-\boldsymbol{u}_{2}$ we get

$$
\begin{aligned}
\int_{\Omega} \partial_{t} \boldsymbol{w}(t) \cdot \boldsymbol{w}(t)+\int_{\Omega}\left(\mathbf{S}\left(\mathbf{D} \boldsymbol{u}_{1}(t)\right)-\mathbf{S}\left(\mathbf{D} \boldsymbol{u}_{2}(t)\right): \mathbf{D} \boldsymbol{w}(t)\right. & \\
\leq \int_{\Omega}\left(\boldsymbol{u}_{1}(t) \otimes \boldsymbol{u}_{1}(t)-\boldsymbol{u}_{2}(t) \otimes\right. & \left.\boldsymbol{u}_{2}(t)\right): \nabla \boldsymbol{w}(t) \\
& \quad+\int_{\Omega}\left(\boldsymbol{f}_{1}-\boldsymbol{f}_{2}\right) \cdot \boldsymbol{w}(t)+\Theta(t)+\Upsilon(t),
\end{aligned}
$$

where

$$
\begin{aligned}
\Theta(t):=\int_{\Omega} \partial_{t} \boldsymbol{u}_{1}(t) \cdot\left(\boldsymbol{u}_{12}(t)-\boldsymbol{u}_{2}(t)\right)+\int_{\Omega} \partial_{t} \boldsymbol{u}_{2}(t) \cdot\left(\boldsymbol{u}_{21}(t)-\boldsymbol{u}_{1}(t)\right) \\
+\int_{\Omega} \mathbf{S}\left(\mathbf{D} \boldsymbol{u}_{1}(t)\right): \mathbf{D}\left(\boldsymbol{u}_{12}(t)-\boldsymbol{u}_{2}(t)\right)+\int_{\Omega} \mathbf{S}\left(\mathbf{D} \boldsymbol{u}_{2}(t)\right): \mathbf{D}\left(\boldsymbol{u}_{21}(t)-\boldsymbol{u}_{1}(t)\right) \\
\quad+\int_{\Omega} \boldsymbol{f}_{1} \cdot\left(\boldsymbol{u}_{2}(t)-\boldsymbol{u}_{12}(t)\right)+\int_{\Omega} \boldsymbol{f}_{2} \cdot\left(\boldsymbol{u}_{1}(t)-\boldsymbol{u}_{21}(t)\right)
\end{aligned}
$$

and

$$
\Upsilon(t):=\int_{\Omega}\left(\boldsymbol{u}_{1}(t) \otimes \boldsymbol{u}_{1}(t)\right): \nabla\left(\boldsymbol{u}_{2}(t)-\boldsymbol{u}_{12}(t)\right)+\int_{\Omega}\left(\boldsymbol{u}_{2}(t) \otimes \boldsymbol{u}_{2}(t)\right): \nabla\left(\boldsymbol{u}_{1}(t)-\boldsymbol{u}_{21}(t)\right) .
$$

The estimates (2.8) and (2.9) and the structural condition (1.5a) allow us to conclude that there exists a positive constant $C$ such that, for any $t \in(0, T)$,

$$
\int_{0}^{t}(\Theta(\tau)+\Upsilon(\tau)) \leq C\left\|\psi_{1}-\psi_{2}\right\|_{L^{\infty}\left(Q_{T}\right)}
$$

Integrating (3.2) in time, using (2.8), (2.12) and (3.3) we obtain

$$
\begin{aligned}
\|\boldsymbol{w}(t)\|_{\boldsymbol{L}^{2}(\Omega)}^{2}+C_{1} \int_{0}^{t}\|\mathbf{D} \boldsymbol{w}\|_{\boldsymbol{L}^{2}(\Omega)}^{2} \leq C_{2} \int_{0}^{t}\|\boldsymbol{w}\|_{\boldsymbol{L}^{2}(\Omega)}\|\mathbf{D} \boldsymbol{w}\|_{\boldsymbol{L}^{2}(\Omega)} \\
\quad+C_{3} \int_{0}^{t}\left\|\boldsymbol{f}_{1}-\boldsymbol{f}_{2}\right\|_{\boldsymbol{L}^{2}(\Omega)}\|\boldsymbol{w}\|_{\boldsymbol{L}^{2}(\Omega)}+\|\boldsymbol{w}(0)\|_{\boldsymbol{L}^{2}(\Omega)}^{2}+C_{4}\left\|\psi_{1}-\psi_{2}\right\|_{L^{\infty}\left(Q_{T}\right)}
\end{aligned}
$$

and so,

$$
\|\boldsymbol{w}(t)\|_{\boldsymbol{L}^{2}(\Omega)}^{2} \leq C_{5} \int_{0}^{t}\|\boldsymbol{w}\|_{\boldsymbol{L}^{2}(\Omega)}^{2}+C_{6}\left(\left\|\boldsymbol{f}_{1}-\boldsymbol{f}_{2}\right\|_{\boldsymbol{L}^{2}\left(Q_{T}\right)}^{2}+\|\boldsymbol{w}(0)\|_{\boldsymbol{L}^{2}(\Omega)}^{2}+\left\|\psi_{1}-\psi_{2}\right\|_{L^{\infty}\left(Q_{T}\right)}\right) .
$$

By the integral Gronwall's inequality we conclude that there exists a positive constant $C$, depending on $T$, such that

$$
\|\boldsymbol{w}\|_{L^{\infty}\left(0, T ; \boldsymbol{L}^{2}(\Omega)\right)}^{2} \leq C\left(\left\|\boldsymbol{f}_{1}-\boldsymbol{f}_{2}\right\|_{\boldsymbol{L}^{2}\left(Q_{T}\right)}^{2}+\|\boldsymbol{w}(0)\|_{\boldsymbol{L}^{2}(\Omega)}^{2}+\left\|\psi_{1}-\psi_{2}\right\|_{L^{\infty}\left(Q_{T}\right)}\right) .
$$

Returning to (3.4), the last inequality allows us to conclude (3.1).

Given

$$
\boldsymbol{f}_{\infty} \in \boldsymbol{L}^{1}(\Omega), \quad \psi_{\infty} \in L^{\infty}(\Omega) \text { such that } \psi_{\infty}(x) \geq \psi_{*}>0 \text { a.e in } \Omega,
$$

and setting the convex set

$$
\mathbb{K}_{\infty}:=\left\{\boldsymbol{w} \in \mathbb{V}^{q}:|\mathbf{D} \boldsymbol{w}| \leq \psi_{\infty}, \text { a.e. in } \Omega\right\},
$$

we consider now the stationary variational inequality: Find $\boldsymbol{u}_{\infty} \in \mathbb{K}_{\infty}$, such that,

$$
\int_{\Omega} \mathbf{S}(\mathbf{D} \boldsymbol{u}): \mathbf{D}(\boldsymbol{w}-\boldsymbol{u})-\int_{\Omega}(\boldsymbol{u} \otimes \boldsymbol{u}): \nabla(\boldsymbol{w}-\boldsymbol{u}) \geq \int_{\Omega} \boldsymbol{f} \cdot(\boldsymbol{w}-\boldsymbol{u}), \quad \forall \boldsymbol{w} \in \mathbb{K}_{\infty}
$$


Theorem 3.2. Under the assumptions (3.5) the steady-state variational inequality (3.6) has a solution $\boldsymbol{u}_{\infty} \in$ $\mathbb{K}_{\infty} \cap \mathscr{C}^{0, \gamma}(\bar{\Omega})^{d}, 0 \leq \gamma<1$. Moreover, there exists a constant $\mu_{*}>0$, such that, if $\mu>\mu_{*}$ in $(1.5 \mathrm{~b})$, the solution is unique.

Proof. The existence of a solution follows by the general theory for pseudo-monotone operators exactly as in [15].

Considering two solutions of the problem, $\boldsymbol{u}_{\infty 1}$ and $\boldsymbol{u}_{\infty 2}$, denoting $\boldsymbol{w}_{\infty}=\boldsymbol{u}_{\infty 1}-\boldsymbol{u}_{\infty 2}$, the following inequality is satisfied

$$
\int_{\Omega}\left(\mathbf{S}\left(\mathbf{D} \boldsymbol{u}_{\infty 1}\right)-\mathbf{S}\left(\mathbf{D} \boldsymbol{u}_{\infty 2}\right)\right): \mathbf{D}\left(\boldsymbol{w}_{\infty}\right) \leq \int_{\Omega}\left(\boldsymbol{u}_{\infty 1} \otimes \boldsymbol{u}_{\infty 1}-\boldsymbol{u}_{\infty 2} \otimes \boldsymbol{u}_{\infty 2}\right): \nabla \boldsymbol{w}_{\infty}
$$

By $(1.5 \mathrm{~b})$, there exists a positive constant $C_{1}$ such that

$$
\int_{\Omega}\left(\mathbf{S}\left(\mathbf{D} \boldsymbol{u}_{\infty 1}\right)-\mathbf{S}\left(\mathbf{D} \boldsymbol{u}_{\infty 2}\right)\right): \mathbf{D}\left(\boldsymbol{w}_{\infty}\right) \geq \mu C_{1}\left\|\mathbf{D} \boldsymbol{w}_{\infty}\right\|_{\boldsymbol{L}^{2}(\Omega)}^{2}
$$

and, as in (2.12), using Poincaré and Korn inequalities,

$$
\left|\int_{\Omega}\left(\boldsymbol{u}_{\infty 1} \otimes \boldsymbol{u}_{\infty 1}-\boldsymbol{u}_{\infty 2} \otimes \boldsymbol{u}_{\infty 2}\right): \nabla \boldsymbol{w}_{\infty}\right| \leq C_{2}\left\|\boldsymbol{w}_{\infty}\right\|_{\boldsymbol{L}^{2}(\Omega)}\left\|\mathbf{D} \boldsymbol{w}_{\infty}\right\|_{\boldsymbol{L}^{2}(\Omega)} \leq C_{3}\left\|\mathbf{D} \boldsymbol{w}_{\infty}\right\|_{\boldsymbol{L}^{2}(\Omega)}^{2} .
$$

The last three inequalities leads to

$$
\left(\mu C_{1}-C_{3}\right)\left\|\mathbf{D} \boldsymbol{w}_{\infty}\right\|_{\boldsymbol{L}^{2}(\Omega)} \leq 0
$$

and so, if $\mu>\frac{C_{3}}{C_{1}}$, we have uniqueness.

Remark 3.3. We observe that, as in the stationary Navier-Stokes equation (see, for instance, [8, p. 118]) we have uniqueness of the solution for large viscosities (or low Reynold's numbers), but here the lower bound $\mu_{*}=\frac{C_{3}}{C_{1}}$ may be calculated in terms of the $L^{\infty}$ norm of the data $\psi_{\infty}$, in order to control the constants $C_{3}$ and $C_{1}$. Note that, by (1.5b), $C_{1}=1$ if $q=2$ but if $1<q<2$ or $q>2$ we could not find a similar uniqueness result in the literature even for the corresponding equation.

Theorem 3.4. Let $\boldsymbol{u}=\boldsymbol{u}(t)$ be the solution of problem (2.4) with $T=\infty$ under the assumptions

$$
\boldsymbol{f} \in L^{\infty}\left(0, \infty ; \boldsymbol{L}^{q^{\prime} \vee 2}(\Omega)\right), \quad \psi \in W^{1, \infty}\left(0, \infty ; L^{\infty}(\Omega)\right) \quad \text { and } \quad \psi \geq \psi^{*}>0
$$

and let $\boldsymbol{u}_{\infty}$ be the unique solution of problem (3.6), under the assumptions (3.5) by assuming there exists a constant $\mu_{*}>0$, such that, if $\mu>\mu_{*}$ in (1.5b).

Suppose also that there exists $\delta>\frac{1}{2}$, such that, for $t>t_{0}$

$$
\beta(t):=\left\|\psi(t)-\psi_{\infty}\right\|_{L^{\infty}(\Omega)} \leq \frac{1}{t^{\delta}}
$$

and

$$
\varphi(t):=\int_{t}^{t+1} \int_{\Omega}\left|\boldsymbol{f}(\tau)-\boldsymbol{f}_{\infty}\right| d \tau \underset{t \rightarrow \infty}{\longrightarrow} 0
$$

Then

$$
\zeta(t):=\left\|\boldsymbol{u}(t)-\boldsymbol{u}_{\infty}\right\|_{\boldsymbol{L}^{2}(\Omega)}^{2} \underset{t \rightarrow \infty}{\longrightarrow} 0 .
$$

Proof. Denoting $\rho(t):=\frac{\lambda_{*}}{\lambda_{*}+\beta(t)}$ and arguing as in the proof of Theorem 3.1, we set $\boldsymbol{w}_{\infty}=\rho(t) \boldsymbol{u}(t) \in \mathbb{K}_{\infty}$ in (3.6) and $\boldsymbol{w}=\rho(t) \boldsymbol{u}_{\infty} \in \mathbb{K}(t)$ in (2.4). Denoting $\boldsymbol{v}(t):=\boldsymbol{u}(t)-\boldsymbol{u}_{\infty}$, we obtain

$$
\begin{array}{r}
\int_{\Omega} \partial_{t} \boldsymbol{u}(t) \cdot \boldsymbol{v}(t)+\int_{\Omega}\left(\mathbf{S}(\mathbf{D} \boldsymbol{u}(t))-\mathbf{S}\left(\mathbf{D} \boldsymbol{u}_{\infty}\right)\right): \mathbf{D} \boldsymbol{v}(t) \\
\leq \int_{\Omega}\left(\boldsymbol{f}(t)-\boldsymbol{f}_{\infty}\right) \cdot \boldsymbol{v}(t)+\rho(t) \int_{\Omega}\left(\left(\boldsymbol{u}_{\infty} \otimes \boldsymbol{u}_{\infty}\right): \nabla \boldsymbol{u}(t)-\left(\boldsymbol{u}(t) \otimes \boldsymbol{u}(t): \nabla \boldsymbol{u}_{\infty}\right)\right) \\
+(1-\rho(t)) \int_{\Omega} \partial_{t} \boldsymbol{u}(t) \cdot \boldsymbol{u}_{\infty}+\Theta(t)
\end{array}
$$


where

$$
\Theta(t):=(1-\rho(t)) \int_{\Omega}\left|\mathbf{S}(\mathbf{D} \boldsymbol{u}(t)): \mathbf{D} \boldsymbol{u}_{\infty}-\mathbf{S}\left(\mathbf{D} \boldsymbol{u}_{\infty}\right): \mathbf{D} \boldsymbol{u}(t)+\boldsymbol{f}_{\infty} \cdot \boldsymbol{u}(t)-\boldsymbol{f}(t) \cdot \boldsymbol{u}_{\infty}\right|
$$

Using the properties of $\mathbf{S}$, as in (2.12), we obtain from (3.9)

$\frac{d}{d t}\|\boldsymbol{v}(t)\|_{\boldsymbol{L}^{2}(\Omega)}^{2}+\mu C_{1}\|\mathbf{D} \boldsymbol{v}(t)\|_{\boldsymbol{L}^{2}(\Omega)}^{2} \leq C_{2}\left\|\boldsymbol{f}(t)-\boldsymbol{f}_{\infty}\right\|_{\boldsymbol{L}^{1}(\Omega)}+C_{3}\|\mathbf{D} \boldsymbol{v}(t)\|_{\boldsymbol{L}^{2}(\Omega)}^{2}+C_{4} \beta(t)\left\|\partial_{t} \boldsymbol{u}(t)\right\|_{\boldsymbol{L}^{2}(\Omega)}+2 \Theta(t)$

As we are assuming that $\mu$ is large enough, we obtain, applying Poincaré and Korn inequalities, that

$$
\frac{d}{d t}\|\boldsymbol{v}(t)\|_{\boldsymbol{L}^{2}(\Omega)}^{2}+C\|\boldsymbol{v}(t)\|_{\boldsymbol{L}^{2}(\Omega)}^{2} \leq \Upsilon(t)
$$

where

$$
\Upsilon(t):=C_{2}\left\|\boldsymbol{f}(t)-\boldsymbol{f}_{\infty}\right\|_{\boldsymbol{L}^{1}(\Omega)}+C_{4} \beta(t)\left\|\partial_{t} \boldsymbol{u}(t)\right\|_{\boldsymbol{L}^{2}(\Omega)}+2 \Theta(t)
$$

By the proof of estimate (2.9) we conclude that there exists a constant $D$, independent of $t \geq 1$ such that

$$
\left\|\partial_{t} \boldsymbol{v}\right\|_{L^{2}\left(Q_{t}\right)}^{2} \leq D t
$$

so, assumptions (3.7) and (3.8) leads to

$$
\int_{t}^{t+1} \Upsilon(\tau) d \tau \underset{t \rightarrow \infty}{\longrightarrow} 0
$$

By a well-known result (see, for instance [6, p. 268]), we may conclude

$$
\zeta(t)=\|\boldsymbol{v}(t)\|_{\boldsymbol{L}^{2}(\Omega)}^{2} \leq e^{-\frac{C t}{2}}+\frac{1}{1-e^{-C}} \sup _{s \geq \frac{t}{2}} \int_{s}^{s+1} \Upsilon(\tau) d \tau \underset{t \rightarrow \infty}{\longrightarrow} 0 .
$$

\section{Acknowledgment}

The research was partially supported by CMAT at the University of Minho with the Portuguese Funds from FCT, through the Project PEstOE/MAT/UI0013/2014, as well as by the CMAF/FCUL at the University of Lisbon.

\section{References}

[1] H.A. Barnes, Shear-Thickening ("Dilatancy") in Suspensions on Nonaggregating Solid Particles Dispersed in Newtonian Liquids, J. Rheology, 33 (2) (1989), 329-366.

[2] H. Beirão da Veiga, Navier-Stokes Equations with Shear-Thickening Viscosity. Regularity up to the Boundary, J. Math. Fluid Mech. 11 (2009), 233-257.

[3] J.C. De los Reyes, G. Stadler, A nonsmooth model for discontinuous shear thickening fluids: analysis and numerical solution, Interfaces Free Bound. 16 (4) (2014), 575-602.

[4] L. Diening, M. Ruzicka, J. Wolf, Existence of weak solutions for unsteady motions of generalized Newtonian fluids, Ann. Sc. Norm. Super. Pisa Cl. Sci. (5) 9 (1) (2010), 1-46.

[5] G. Duvaut, J.L. Lions, Les Inéquations en Mécanique et en Physique, Dunod, Paris, 1972.

[6] A. Haraux, Nonlinear Evolution Equations. Global behavior of solutions, Lect. Notes in Math. 841, Springer-Verlag, 1981. 
[7] O.A. Ladyzhenskaya, New equations for the description of the motions of viscous incompressible fluids, and global solvability for their boundary value problems, Boundary value problems of mathematical physics, Part 5, Trudy Mat. Inst. Steklov 102 (1967), 85-104.

[8] O.A. Ladyzhenskaya, The Mathematical Theory of Viscous Incompressible Flow, 2nd ed., Gordon and Breach, New York, 1969.

[9] Y.S. Lee, E.D. Wetzel, N.J. Wagner, The ballistic impact characteristics of Kevlar woven fabrics impregnated with a colloidal shear thickening fluid, J. Materials Sci. 38 (2004), 2825-2833.

[10] J.L. Lions, Quelques Methodes de Resolution des Problemes aux Limites Non Lineaires, Dunod, Paris, 1969.

[11] J. Málek, J. Nečas, M. Rokyta, M. and M. Růžička, Weak and measure-valued solutions to evolutionary PDEs. Chapman \& Hall, London, 1996.

[12] J. Malek, K.R. Rajagopal, Mathematical issues concerning the Navier-Stokes equations and some of its generalizations, in Evolutionary equations. Vol. II, Handb. Differ. Equ., 371-459, Elsevier/North-Holland, Amsterdam, 2005.

[13] F. Miranda, J.F. Rodrigues, L. Santos, On a p-curl system arising in electromagnetism, Discrete Contin. Dyn. Syst. Ser. S 5, no. 3, (2012), 605-629.

[14] L. Prigozhin, Sandpiles and river networks: extended systems with non-local interactions, Phys. Rev. E 49 (1994), 1161-1167.

[15] J.F. Rodrigues, On the Mathematical Analysis of Thick Fluids, in Boundary-value problems of mathematical physics and related problems of function theory. Part 44, Zap. Nauchn. Sem. POMI 425, 117-136, 2014.

[16] J.F. Rodrigues, L. Santos, Quasivariational solutions for first order quasilinear equations with gradient constraint, Ann. Scuola Norm. Pisa Cl. Sci. 29 (2000), 153-169.

[17] J.F. Rodrigues, L. Santos, A parabolic quasivariational inequality arising in a superconductivity model, Arch. Ration. Mech. Anal. 205 (2) (2012), 493-514.

[18] L. Santos, Variational problems with non-constant gradient constraints, Portugaliae Math. 59 (2002), 205-248.

[19] S.A. Sazhenkov, The Problem of Motion of Rigid Bodies in a Non-Newtonean Incompressible Fluid, Siberian Math. J. 39 (1998), 126-140.

[20] J. Simon, Compact sets in the space $L^{p}(0, T ; B)$, Ann. Mat. Pura Appl. (4) 146, (1987), 65-96.

[21] V.V. Shelukhin, Bingham viscoplastic as a limit of non-Newtonian fluids, J. Math. Fluid Mech. 4 (2) (2002), 109-127.

[22] N.J. Wagner, J.F. Brady, Shear thickening in colloidal dispersions, Physics Today (62) (10) 2009, 27-32. 